\title{
The Effect of Different Strategies and the Structure on Opinion Formation
}

\author{
Cheng Ju, ${ }^{1}$ Yang Cao, ${ }^{2}$ and Pengcheng Zhou ${ }^{1}$ \\ ${ }^{1}$ Department of Mathematics, Southeast University, Nanjing 210096, China \\ ${ }^{2}$ College of Telecommunications and Information Engineering, Nanjing University of Posts and Telecommunications, \\ Nanjing 210023, China
}

Correspondence should be addressed to Cheng Ju; jucheng1992@qq.com

Received 20 January 2013; Revised 14 May 2013; Accepted 14 May 2013

Academic Editor: Yong Zhou

Copyright (C) 2013 Cheng Ju et al. This is an open access article distributed under the Creative Commons Attribution License, which permits unrestricted use, distribution, and reproduction in any medium, provided the original work is properly cited.

\begin{abstract}
This paper defines two different ways of the process of the opinion formation and focuses on the effect of the proportion of the two strategies of process and the structure of the network. A multiopinion model is proposed in this paper, which includes two strategies of opinion formation. At the first part, the change of the structured network and the change of the single node are used as two strategies of the process of the opinion formation. We focus on how the proportion of the two strategies can affect the outcome of the process. At the second part, as the proportion of the two strategies is fixed, the edges are dense in the community and sparse outside. Thus we can construct a bifurcation diagram to be verified through experimental study. The phase transition is studied in the network which contains more than four opinions and two strategies of process. Our results show that the size of the group and the dense of edges are the two important features for the process of opinion formation.
\end{abstract}

\section{Introduction}

In the field of social networks analysis, the study of opinions dynamics has attracted a growing amount of work $[1,2]$. To describe some collective behaviors in both human societies and social organization, several useful models have been provided. In social network modeling, various networks have gradually been proposed. The model developed from the simplest full connected networks, Euclidean geometry in one, two or more dimensions, to very advanced network topologies [3-5]. The application of Ising models and other tools of statistical or computational physics to opinion formation have a long tradition from the work of di Ettore Majorana [6]. Some other models such as those by Boccaletti et al. are reviewed in [7]. The network models are much better at representing the actual relationships between people and groups.

One of the most important properties for the social networks is the community structure [8]. It means that the vertices within the groups have higher density of edges while vertices among groups have lower density of edges $[9,10]$. The community structure appears to be common to many networks reflected by the well-researched scientific collaboration network [11, 12], the Internet, the World Wide Web, and so forth. Similarly, the vertices can also be divided into different groups because of their own properties and the relationship with each other in power grids [13], food web, and biological networks [14]. In the fields of physics, sociology, and so forth, community detection in networks also becomes a hot research topic $[8,15]$. To mimic the existence of communities in social networks in the field of opinion formation, Lambiotte et al. analyze the opinions dynamics in a topology consisting of two coupled fully connected networks [16]. It focused on the transition behavior at the value of the interconnectivity parameter. Huang et al. also focused on the effect of some temperature-like parameters as the social impact model $[17,18]$. Moreover, Qian et al. discussed the adaptive bridge control strategy for opinion evolution on social networks [19].

In order to understand the effect of the community structure and the different strategies of opinion process in the opinions dynamics, for simplicity, a multiopinion model is proposed in this paper, including two strategies of 
TABle 1: Symbol table.

\begin{tabular}{ll}
\hline Symbol & Description \\
\hline$N$ & The number of nodes in the entire network \\
$M$ & The number of opinions \\
$K$ & The number of nodes of the small community \\
$L$ & The average number of edges for every node \\
$P$ & The number of edges for the nodes in the small \\
& community \\
$t$ & The proportion of the first way of the process \\
$O x$ & Time (or the number of the steps) \\
$\alpha, \beta, \phi, \varphi, \theta, \ldots$ & The opinion of node $x$ \\
$n_{x \alpha}$ & The number of nodes that have the opinion $\alpha$ and \\
$n_{x}$ & connect to the node $x$ \\
$\rho$ & The number of nodes which connect to the node $x$ \\
\hline
\end{tabular}

opinion formation. It does not aim at an exact description of reality. However, it focuses on discovering some essential and fundamental features of an otherwise very complex and multiple phenomena by doing some crude approximations. Therefore, we use Monte Carlo method to simulate the reality and determine the sensitivity of the model in the different situation.

In this paper, to understand the mechanism of the community structure in the opinions dynamics, we focus on the following four questions.

(1) How can the different proportion of the process of the opinion formation affect the dynamic of the model? At which proportion the model is most sensitive to the change of the proportion?

(2) How can the size of the community contribute to the outcome of the model when the proportion of the process is fixed?

(3) Could the community structure affect the evolution of the network?

(4) How can different kinds of the noise contribute to the evolution of the network?

In the first part of this paper, we investigate the outcome of the model with different ways of the process of the opinion formation. The first way of transition of the opinion is the change of the structure of the network and the second way is the intersecting opinion of the different points in the network. The different proportion of the ways of processing will lead to the different outcome of the dynamic model.

If the model only uses the first way, the opinion of every node will not change, while the structure of the network will change. Hence, at the final phase of the processing, the entire network will evolute into several small communities and there should be no intersects between two different networks.

If the model only uses the second way, the opinion of different nodes will change while the structure will remain the same. In this situation, the model will develop into single network where the structure remains the same.
First of all, we define the term "win," that is, if one of the several opinions have occupied more than the $95 \%$ of nodes in the network in less than 1000 steps. Using Monte Carlo method, we simulate the situation for 5000 times each for its first 1000 steps. We count the probability of win for every $5 \%$ change of the proportion of the two strategies.

In the second part of this paper, we focus on the last three questions. In the same way, we use Monte Carlo method to simulate the different situation. At first, we define a small community in the network of four different opinions. Then we change the size of the community and find out the relation of the size and the probability for the community to occupy the entire network. Secondly, we fix the size of the community and change the structure of the community. And we discover the relation of the structure of the community and the outcome.

At last, we add two different noises into the model. The first kind of noise is the white noise and the second kind of noise is the linearly decreasing noise. Changing the strength of the noise, we do the same study listed above. Therefore, we can find the role that the noises play in the evolution.

\section{Model Description}

2.1. Symbol Description of Network. Table 1 shows the description of the symbols in our model. At the first part, we consider a network with $N$ nodes and number the node with 1 to $N$. Second, we add $N K / 2$ edges to the node. Then, assume that the four opinions are placed randomly. And each opinion has the same number of nodes.

2.2. Two Strategies of the Process. In the first way of the process, the opinion of every point will not change, while the structure of the network will change in every step. Hence, just like Figure 1, if the node $X$ connects with the node $Y$ and their opinion is different, the node $X$ will remove the edge to the $Y$ and will randomly choose. And the node $X$ will then find a note which has the same opinion to develop a new edge.

The second way of process is the opinions intersected with the different points in the network. We can see from Figure 2 that if there are different opinions among the nodes connecting to the node $x$, it may change into a new opinion. In this process, the node randomly selects one node from the nodes with the same opinion with equal probability.

Determine the probability of the opinion of node $x$ in the next status as

$$
P\left(O_{x}=\alpha \mid P=1\right)=\frac{n_{x \alpha}}{n_{x}},
$$

where $P$ is the proportion of the first way of the process. Hence, $P=1$ means that the first kind of the process is used.

At the second part, we consider a network with $N$ nodes and number the node with 1 to $N$. Then we add $N K / 2$ edges to the node. Consider that the first $M$ node composes a small community $(M<N / 5)$. Furthermore, we assume that other nodes compose three big communities and their sizes are the same.

Then we fix the proportion of two ways of the process and change the size of the small community. 


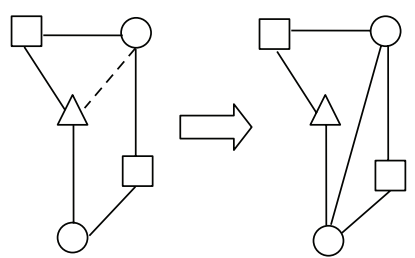

FIGURE 1: Things of one kind come together.

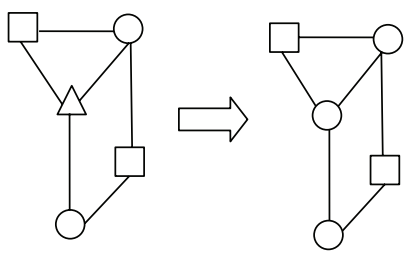

FIGURE 2: One takes the behavior of one's company.

In the following model, we consider $N=1000, Y=5$, $M<200$, and $K=10$.

\subsection{The Description of Noise}

2.3.1. Constant Noise. First, we consider the distant noise.

First, we determine $\varepsilon \sim W N\left(0, \sigma^{2 \prime}\right)$.

Hence, the $\varepsilon$ is distributed in the normal distribution and each $\varepsilon$ is not relevant

Consider the probability of the opinion of node $x$ in the next status when there is constant noise in the model

$$
P\left(O_{x}=\alpha \mid P=1\right)=\frac{n_{X \alpha}}{n_{x}}+\varepsilon,
$$

where $P$ is the proportion of the first way of the process. Hence, $P=1$ means that the first kind of the process is used.

2.3.2. Linear Increasing Noise. Second, we consider the linear increasing noise.

We determine $\varepsilon_{t} \sim W N\left(0, \sigma_{t}^{2 \prime}\right)$.

Hence, although the exception of the noise is still the same, the variance is depending on $t$.

Then, we consider $\sigma_{t}=a+b t$. Therefore, we have the linear noise.

\section{The Sensitivity of the Model to the Proportion of Two Strategies}

3.1. The Outcome of the Model without Noise. In order to determine how the proportion of two ways can affect the model, we first stimulate it without noise. We do experiment every $5 \%$ from $0 \%$ to $100 \%$.

We consider the situation where the $N$ is 800 , and there are four opinions in the network placed randomly on every node. If after 1000 steps one of the opinions can occupy the $95 \%$ of the nodes, we think the outcome is "win."

According to Figure 3, we can see that the probability for win is high when the proportion of first way is little. It means

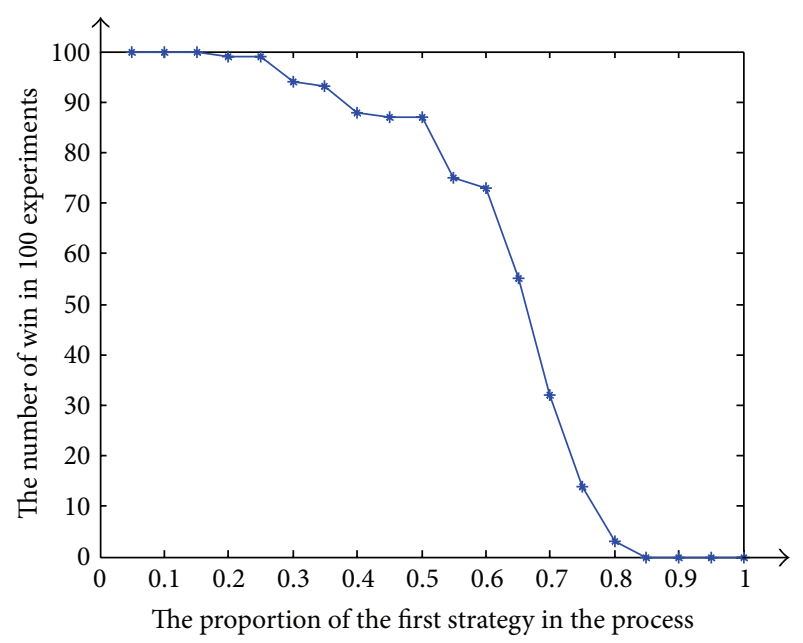

FIgURE 3: The $Y$-ray represents the number of win in 100 experiments. The $X$-ray represents the proportion of two strategies. There is no noise in the model.

that when the proportion of the first way is high, one of the four opinions will occupy the entire network in less than 1000 steps. It can meet our assumption. However, we can see from Figure 3 that the probability for win falls abruptly near the $70 \%$. The most sensitive point is near 0.7 when the proportion is more than $85 \%$, and the probability for win is very small. Moreover, in our experience, when the proportion is more than $90 \%$, no opinion can occupy the entire network and the network is divided into some small communities. Hence, in conclusion, we determine that the phase transition point is 0.7 .

3.2. The Case of Model with Constant Noise. We do some preprocessing to choose the suitable noise for the model. Since $\varepsilon \sim W N\left(0, \sigma^{2 \prime}\right)$, when $\sigma^{2}>5 \%$, it will be difficult to obtain stable results within 1000 steps. Therefore, it may make the model too sensitive. Hence, we choose the $\sigma^{2}=5 \%$.

From Figure 4, compared with the situation without noise, we can find that the model with white noise has some similarities and differences. First, we can see that the probability for win is near $100 \%$ when the proportion is less than $25 \%$. And the probability for win is also decreased by the increase of the proportion. However, unlike the first model, the probability for win decreases after $25 \%$. The change of the slope is less abrupt than the first model.

Remark 1. We do the experiments when $\sigma^{2}=5 \%, \sigma^{2}=$ $7.5 \%$, and $\sigma^{2}=10 \%$ and find that the last two experiments can not get a clear conclusion. Due to the noise, the model cannot get results within 1000 steps. Therefore, we choose $\sigma^{2}=5 \%$. It can be predicted that when $\sigma^{2}$ is near 0 , the curve would tend to the graph without the noise. In the future work, the impact of different values of noise could be used as a research direction.

3.3. The Model with Linear Increasing Noise. We consider $\sigma_{t}=$ $a+b t$. Therefore, we have the linear noise. We want at the 


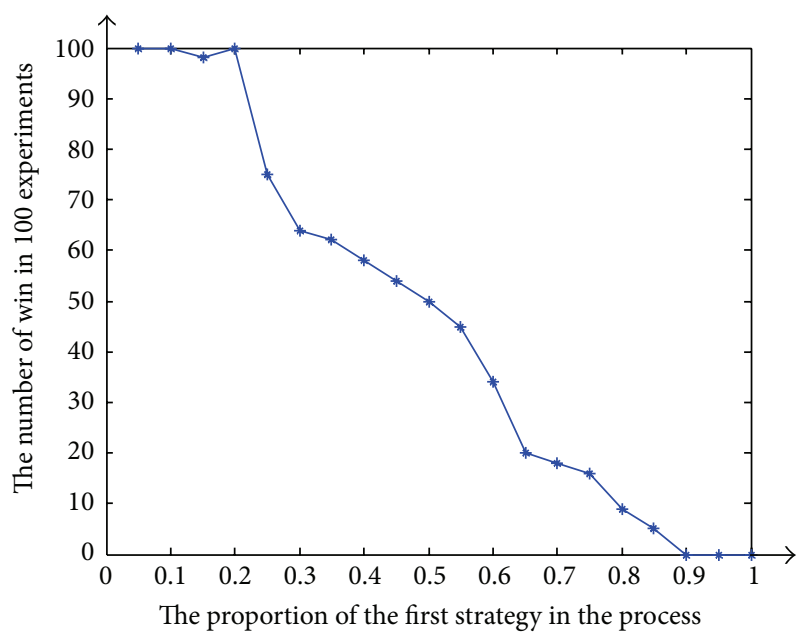

Figure 4: The $Y$-ray represents the number of win in 100 experiments. The $X$-ray represents the proportion of two strategies. There is constant noise in the model.

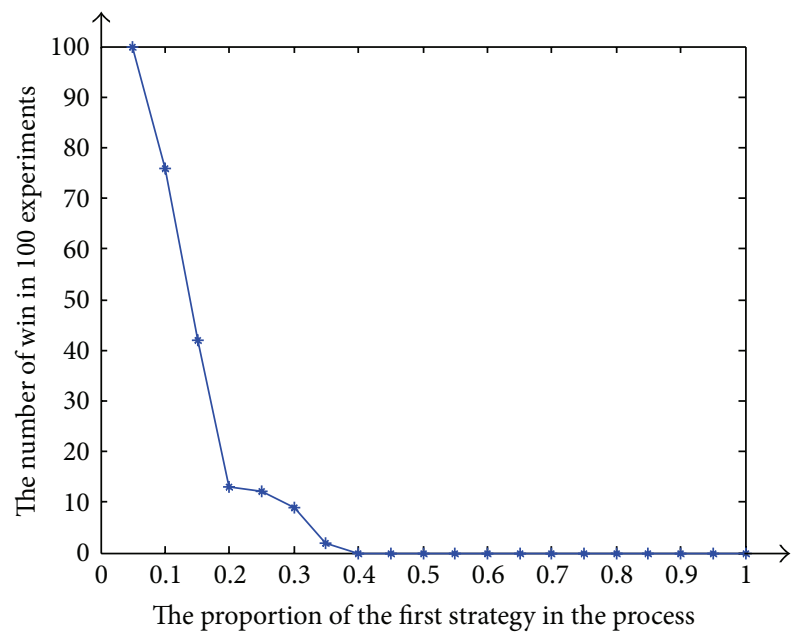

Figure 5: The Y-ray represents the number of win in 100 experiments. The $X$-ray represents the proportion of two strategies. There is linear increasing noise in the model.

beginning the noise to be smaller than the constant noise and when $t=1000$, the noise is more than the constant noise. Hence, we choose $a=3 \%$ and $b=0.005 \%$.

In the linear increasing noise model, we add the noise that increases with the time. It is reasonable because it is our common sense that as the time goes by, it is more difficult for people to have more information about the entire situation and hence they cannot make reasonable decision.

According to Figure 5, we find that it is harder for an opinion to occupy the entire network. And when the proportion is above $45 \%$, no case in our experience that wins will occur. In addition, we find that if one of the opinions cannot occupy most nodes before 500 steps, the win will not occurr because of the increase of the noise.

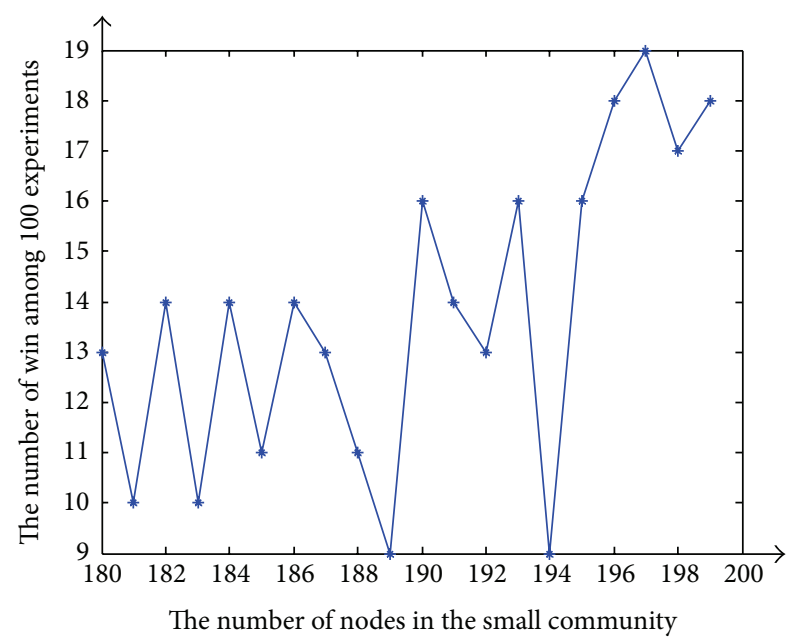

FIgURE 6: The Y-ray represents the number of win among 100 experiments. The $X$-ray represents the number of nodes in the small community. There is no noise in the model.

\section{The Probability for a Community of Minority to Win with the Change of Its Size}

4.1. The Model without Noise. In order to compare with the community with a different structure, we first study the small community in the random network. First, we put 1000 nodes and randomly add 5000 nodes into the network so that $K=5$. We randomly choose $M$ nodes and put opinion $\alpha$ on them so that we can get a small community. Then we randomly put other 4 kinds of other opinions on other nodes outside the small community and do not change the structure of the network after placing the opinions. $M$ is the number of the nodes in the small community. There is no more edge among the community. Therefore, in the following experiments, the $N$ is 1000 and the $K$ is 5 . And we study the relation between $N$ and $M$ with or without noise.

As we can see from Figure 6, when $M=N / 5$, then the probability for the small community to win is near $20 \%$. It is reasonable because the probability for every opinion to win is equal. Then, with the decrease of the nodes in the small community, the probability for it to win falls.

Remark 2. Compared with the formal experiment (the model that only contains two opinions), the probability does not fall dramatically. When the rate of nodes of small community to other community is near $90 \%$, the probability for win is near $12 \%$. However, in the two opinions model, when the rate of size of the small community to others is $90 \%$, the probability for win is 0 . This phenomenon reflects that, in the model that contains more opinions, the influence of $M$ is not so extreme compared with the model of only two opinions.

4.2. The Model with Constant Noise. Then we add noise into the model. When the noise is strong, the probability for every opinion to win is very low. Therefore, in this experiment, we fix the noise with $5 \%$. The probability to win decreases with 


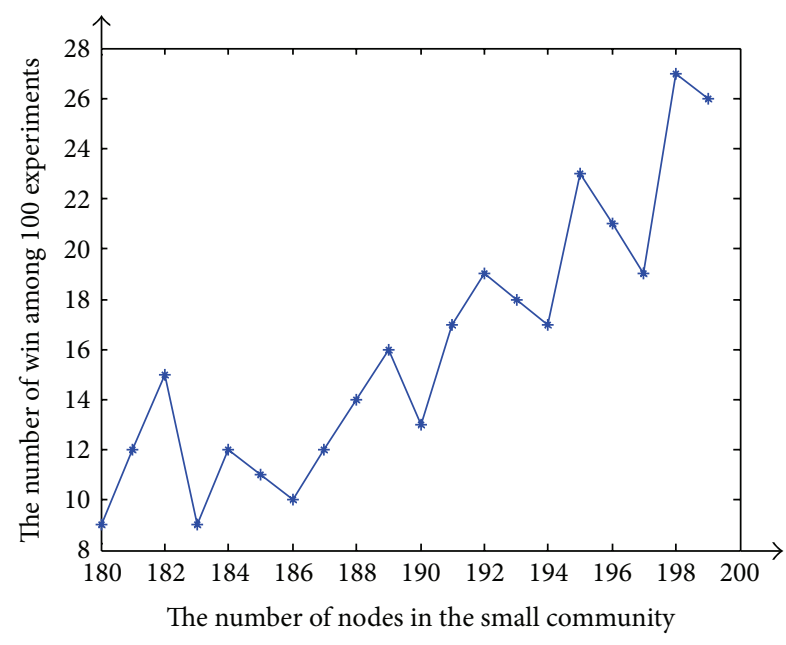

FIGURE 7: The Y-ray represents the number of win among 100 experiments. The $X$-ray represents the number of nodes in the small community. There is constant noise in the model.

the coming of noise. As Figure 7 shows, when the number of nodes in small community is 180 , the probability for win is near $10 \%$. Also, when the number is near 200 , which means the opinion is placed randomly, the probability to win is not stable compared with the last experiment. However, the overall trend is not changed very extreme. Hence, we can see this model have noise community when the noise is not too strong.

\section{The Probability for a Community of Fixed Size to Win with a Different Dense of the Edge}

We determine $M=80, N=1000$, and $K=10$. We add edge into the small community and study the probability for the small community to win.

In the last experiment, we find that when the $L=K=10$, the probability for a small community with 80 nodes to win is about 10. Also we can find from Figure 8, that when the $L-K$ is not big, the probability for the small community to win does not also change perceptively. However, when $L-K>$ 7 , we find that the probability for the small community to win increases abruptly. When the $L=30$, the probability to win is twice as the $L=10$.

In conclusion, when there is no noise, the cohesion also can contribute to the probability for win.

Remark 3. In Figure 9, also do the same experiment when the $M=50$. However, we find that even if the $L-K>20$, the small community cannot win less than 1000 steps. When we make further study in the evolution of the model, we find that the probability for other communities to win is also very small. In other words, the model cannot make an ordered final stage.

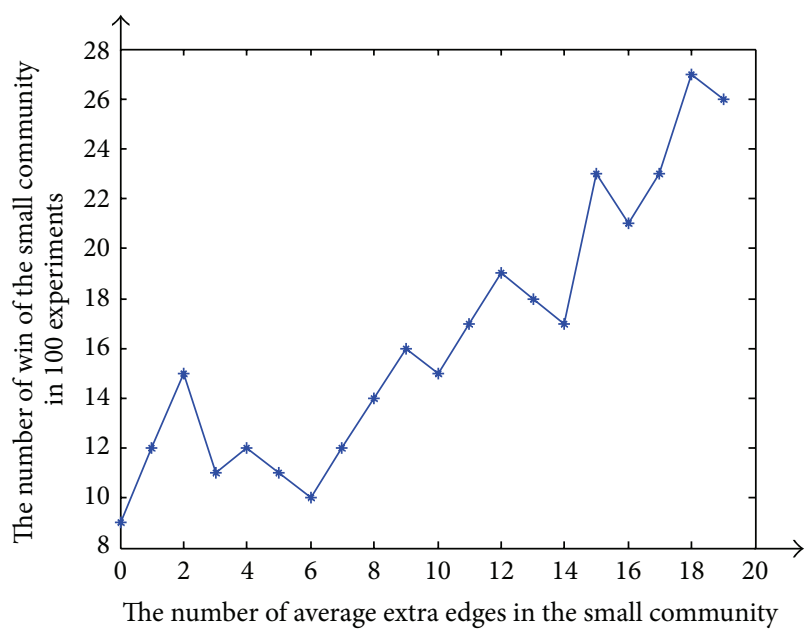

FIGURE 8: The Y-ray represents the number of win of the small community in 100 experiments. The $X$-ray represents the number of average extra edges in the small community. There is no noise in the model and there are 80 nodes in the small community.

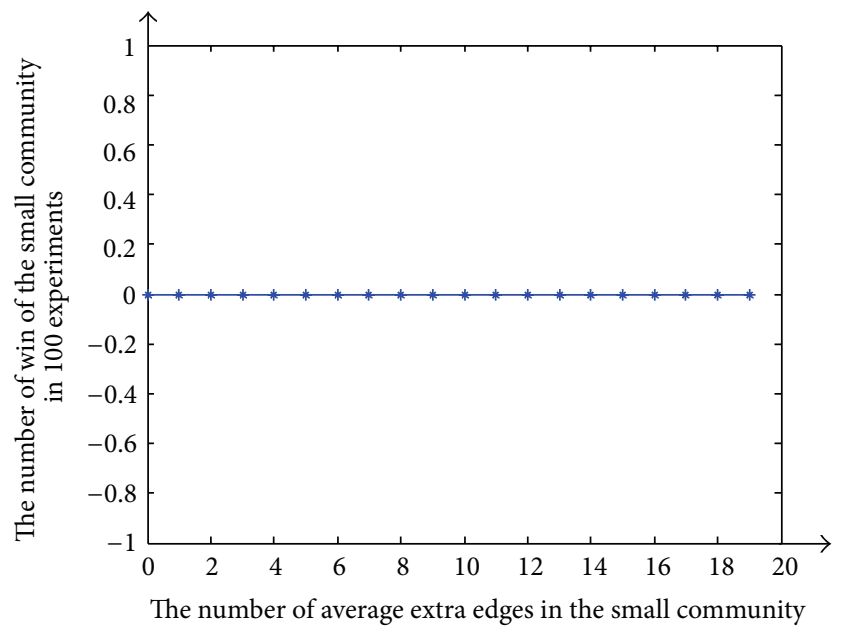

FIGURE 9: The $Y$-ray represents the number of win of the small community among 100 experiments. The $X$-ray represents the number of average extra edges in the small community. There is no noise in the model and there are 50 nodes in the small community.

\section{Conclusions}

Compared with another formal study, the model in this paper focused on the effect of the proportion of two strategies of the opinion formation. Furthermore, we add five different opinions in the model which made the model more complex and more close to the reality. In the experiments, we found the following.

(1) The point of phase transition is near $70 \%$ in the model without noise. Near the proportion of $70 \%$, the probability is strongly sensitive to the change of the proportion of two strategies.

(2) When there are more kinds of opinions in the network, the probability for small community to win is 
less sensitive to the change of its size compared with the model with only two opinions.

(3) The density of edges in the small community can contribute to the probability for it to win. However, it also contributes less compared with the model with only two kinds of opinions.

(4) From the last two remarks, we find that the network with more kinds of opinions is less sensitive to the change of the structure. Hence, it is more stable.

\section{References}

[1] S. Galam, "Real space renormalization group and totalitarian paradox of majority rule voting," Physica A, vol. 285, no. 1, pp. 66-76, 2000.

[2] P. L. Krapivsky and S. Redner, "Dynamics of majority rule in two-state interacting spin systems," Physical Review Letters, vol. 90, no. 23, pp. 238701/1-238701/4, 2003.

[3] M. Boguñá, R. Pastor-Satorras, and A. Vespignani, "Absence of epidemic threshold in scale-free networks with degree correlations," Physical Review Letters, vol. 90, no. 2, pp. 028701/1028701/4, 2003.

[4] M. Lewenstein, A. Nowak, and B. Latané, "Statistical mechanics of social impact," Physical Review A, vol. 45, no. 2, pp. 763-776, 1992.

[5] J. A. Holyst, K. Kacperski, and F. Schweitzer, "Social impact models of opinion dynamics," in Annual Reviews of Computational Physics, D. Stauffer, Ed., vol. 9, pp. 253-273, World Scientific, Singapore, 2001.

[6] N. di Ettore Majorana, "Il valore delle leggi statistiche nella fisica e nelle scienze sociali," Scientia, vol. 36, pp. 55-66, 1942.

[7] S. Boccaletti, V. Latora, Y. Moreno, M. Chavez, and D. U. Hwang, "Complex networks: structure and dynamics," Physics Reports, vol. 424, no. 4-5, pp. 175-308, 2006.

[8] M. Girvan and M. E. J. Newman, "Community structure in social and biological networks," Proceedings of the National Academy of Sciences, vol. 9912, pp. 7821-7826, 2002.

[9] S. Wasserman and K. Faust, Social Network Analysis, Cambridge University Press, New York, NY, USA, 1994.

[10] J. Scott, Social Network Analysis: A Handbook, Sage, Thousand Oaks, Calif, USA, 2000.

[11] M. E. J. Newman, "Scientific collaboration networks. I. Network construction and fundamental results," Physical Review E, vol. 64, no. 1, Article ID 016131, 8 pages, 2001.

[12] M. E. J. Newman, "Scientific collaboration networks. II. Shortest paths, weighted networks, and centrality," Physical Review E, vol. 64, no. 1, Article ID 016132, 7 pages, 2001.

[13] D. J. Watts and S. H. Strogatz, "Collective dynamics of 'smallworld' networks," Nature, vol. 393, no. 6684, pp. 440-442, 1998.

[14] P. Holme, M. Huss, and H. Jeong, "Subnetwork hierarchies of biochemical pathways," Bioinformatics, vol. 19, no. 4, pp. 532538, 2003.

[15] J. Duch and A. Arenas, "Community detection in complex networks using extremal optimization," Physical ReviewE, vol. 72, no. 2, Article ID 027104, 4 pages, 2005.

[16] R. Lambiotte, M. Ausloos, and J. Holyst, "Majority model on a network with communities," Physical Review E, vol. 75, no. 3, Article ID 030101, 4 pages, 2007.

[17] G. Huang, J. Cao, G. Wang, and Y. Qu, "The strength of the minority," Physica A, vol. 387, no. 18, pp. 4665-4672, 2008.
[18] G. Huang, J. Cao, and Y. Qu, “The minority's success under majority rule," Physica A, vol. 388, no. 18, pp. 3911-3916, 2009.

[19] C. Qian, J. Cao, J. Lu, and J. Kurths, "Adaptive bridge control strategy for opinion evolution on social networks," Chaos, vol. 21, no. 2, Article ID 025116, 2011. 


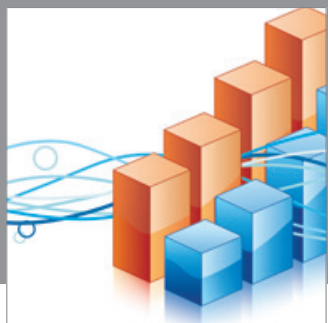

Advances in

Operations Research

mansans

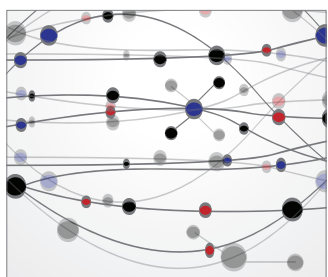

The Scientific World Journal
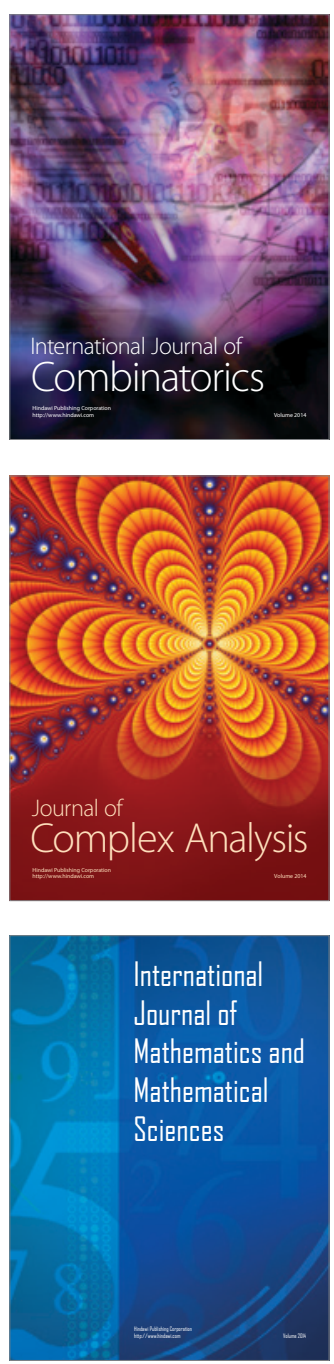
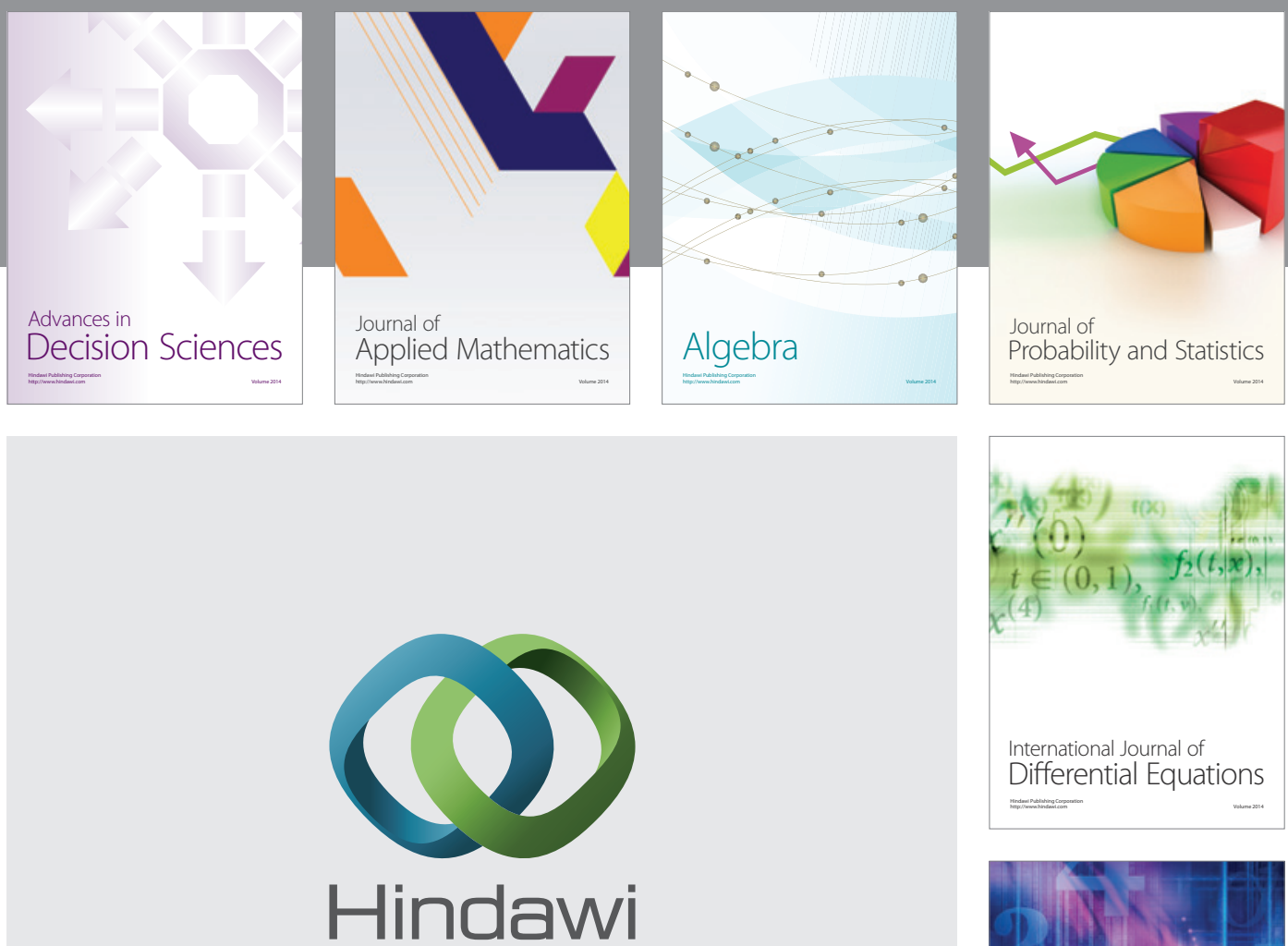

Submit your manuscripts at http://www.hindawi.com
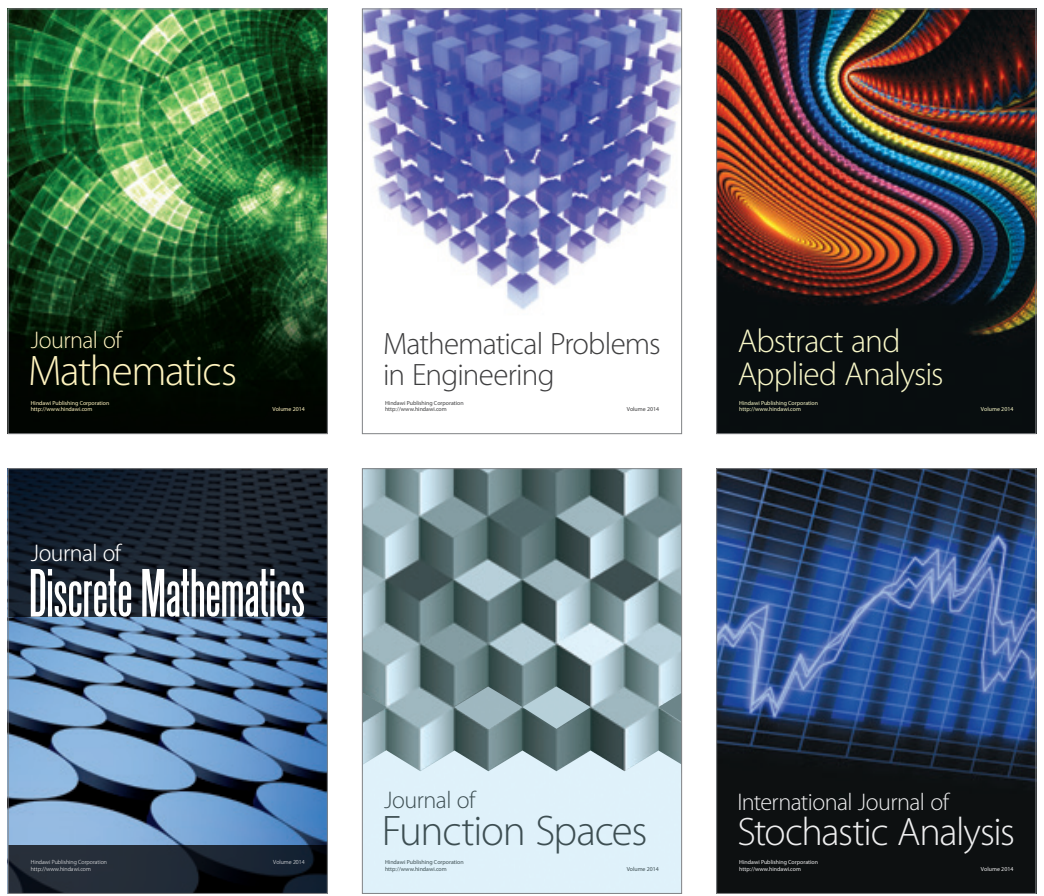

Journal of

Function Spaces

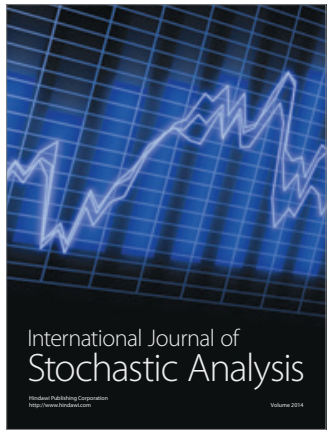

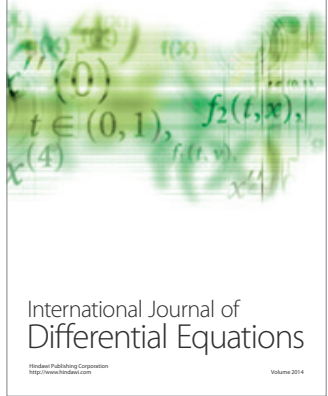
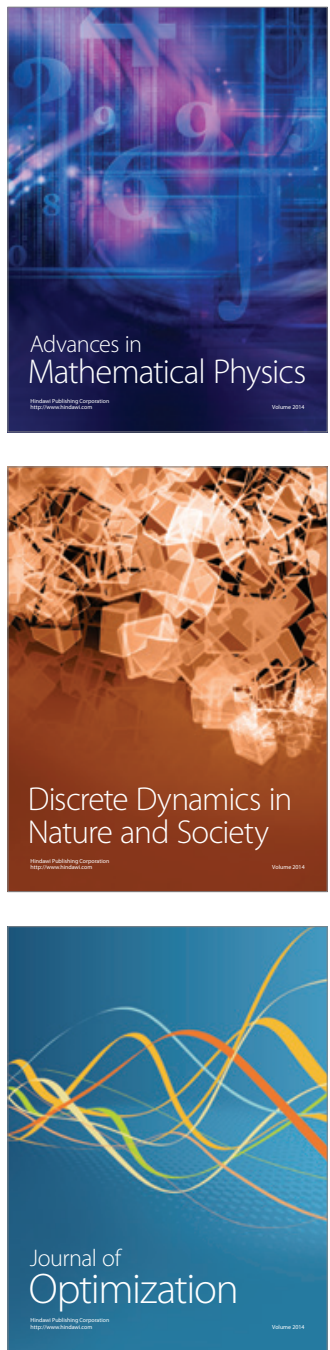